\title{
Ancylostoma duodenale (Dubini, 1843) Creplin, 1843 (Nematoda : Ancylostomidae) parasite de l'hyène tachetée Crocuta crocuta (Erxleben), en Ethiopie
}

\author{
par M. GRABER (*) et J. P. BLANC
}

\begin{abstract}
RESUME
Quelques exemplaires d'Ancylostoma duodenale (Dubini, 1843) Creplin, 1843 ont été recueillis dans l'intestin d'une hyène tachetće abattue dans la province du Harrarghé (Ethiopie).

Ce carnivore, hôte nouveau, semble n'intervenir qu'accidentellement dans l'épidémiologie de l'ancylostomose humaine.
\end{abstract}

Au cours de l'autopsie d'une hyène tachetée adulte, Crocuta crocuta (Erxleben), abattue à Bilen (Moyenne vallée de l'Awash, Province du Harrarghé, Ethiopie), plusieurs parasites ont été recueillis dans le tractus digestif :

- Dans l'estomac, Cylicospirura (Cylicospirura) subaequalis (Molin, 1860), Vevers, 1922 ;

- Dans les dernières portions de l'intestin grêle, Taenia crocutae Mettrick et Beverley-Burton, 1961 et Spirometra pretoriensis Baer, 1924.

Dans le duodénum, ont été isolés des ancylostomidés ( 2 mâles et 5 femelles) que leurs caractères morphologiques et leurs dimensions ne permettent pas de séparer nettement d'Ancylostoma duodenale (Dubini, 1843) Creplin, 1843, parasite qui affecte habituellement l'homme.

Aussi, cette observation présente-t-elle un grand intérêt de par ses implications sur l'épidémiologie de l'ancylostomose humaine.

(*) Chaire de Parasitologie. Ecole Nationale Vétérinaire de Lyon, Marcy l'Etoile, 69260 Charbonnıèresles-Bains.

Mission Vétérinaire Française en Ethiopie, P. O. Box 1053, Addis-Abéba Forestry and Wildlife Authority, P. O. Box 1034, Addis-Abéba.

\section{LE PARASITE}

Le ver est cylindrique, assez épais, de couleur blanchâtre ou légèrement rosée quand il est frais, faiblement atténué à son extrémité antérieure et s'amincissant rapidement au voisinage de l'anus chez la femelle. Il mesure 13-13,6 mm $\times$ $240 \mu\left(\delta^{\star}\right)$ et $15,9-17 \mathrm{~mm} \times 360-380 \mu$ (O).

La cuticule présente une striation transversale avec des stries plus espacées dans la partie moyenne du corps $(6-8 \mu)$ qu'à ses 2 extrémités.

La tête est recourbée vers la face dorsale. La bouche ovalaire donne accès à une capsule buccale infundibuliforme, à parois tourmentées et fortement chitinisées sur son pourtour, dont les dimensions sont les suivantes: hauteur, $180 \mu$ (ठ) à $192 \mu$ (क) ; largeur, 150-156 $\mu$ (d), 150-168 $\mu$ ( $($ ). Elle présente (Pl. 1, fig. 1), sur son bord antérieur et en position ventrale, 2 paires de fortes dents à peine crochues, la dent externe de chaque paire étant un peu plus volumineuse que la dent interne. Parfois, cette dernière est munie d'un petit tubercule ou «troisième dent rudimentaire interne " qu'il n'est pas toujours possible de mettre en évidence, ce qui est le cas pour le matériel examiné. 
Planche 1. - Extrémité antérieure d'Ancylostoma duodenale.

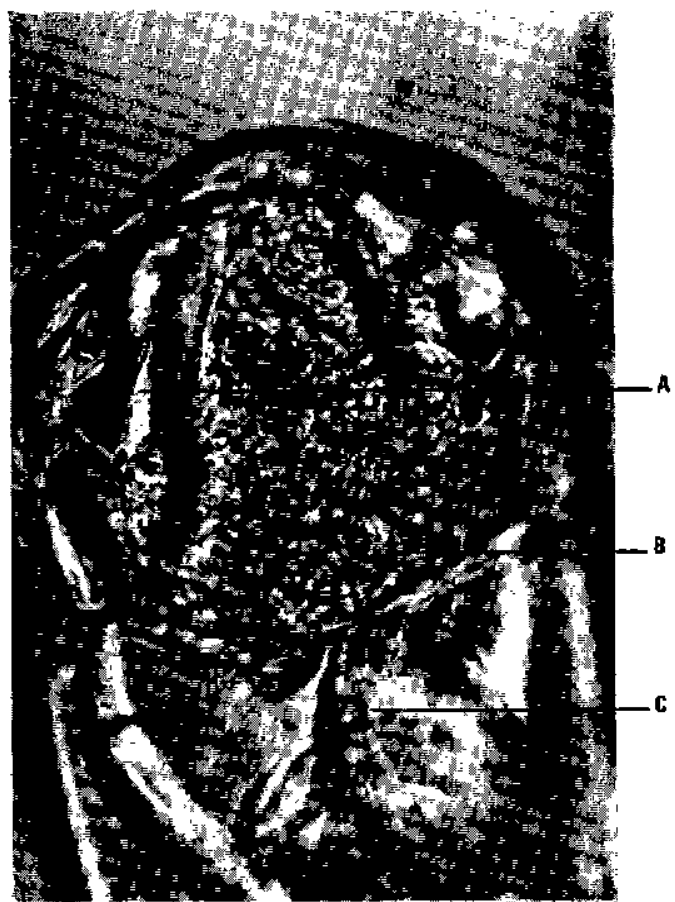

A : Dents ventrales.

B : Incision.

C : Gouttıère dorsale.

Fig. 1. - Capsule buccale.

Vue générale $(x 570)$.

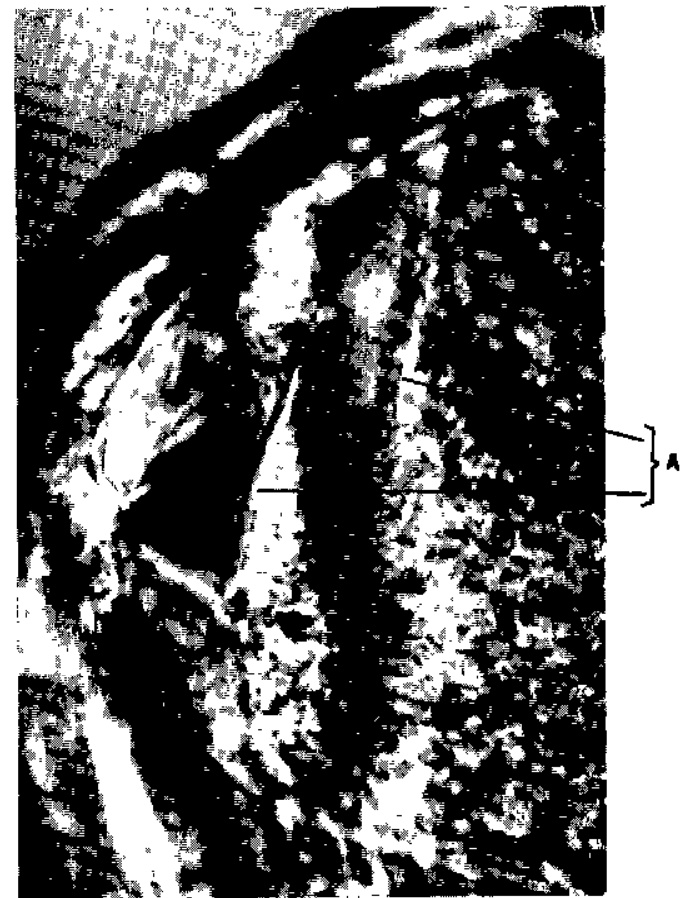

A : Dents ventrales $(1000)$. Fig. 2.

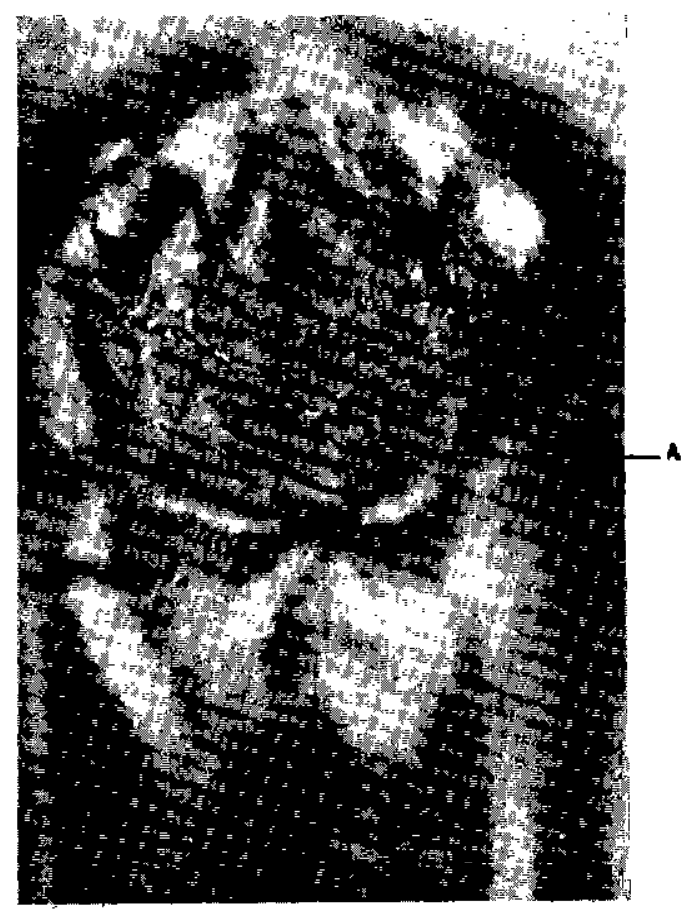

A : Incision ( $\times$ 540),

Fig. 3.

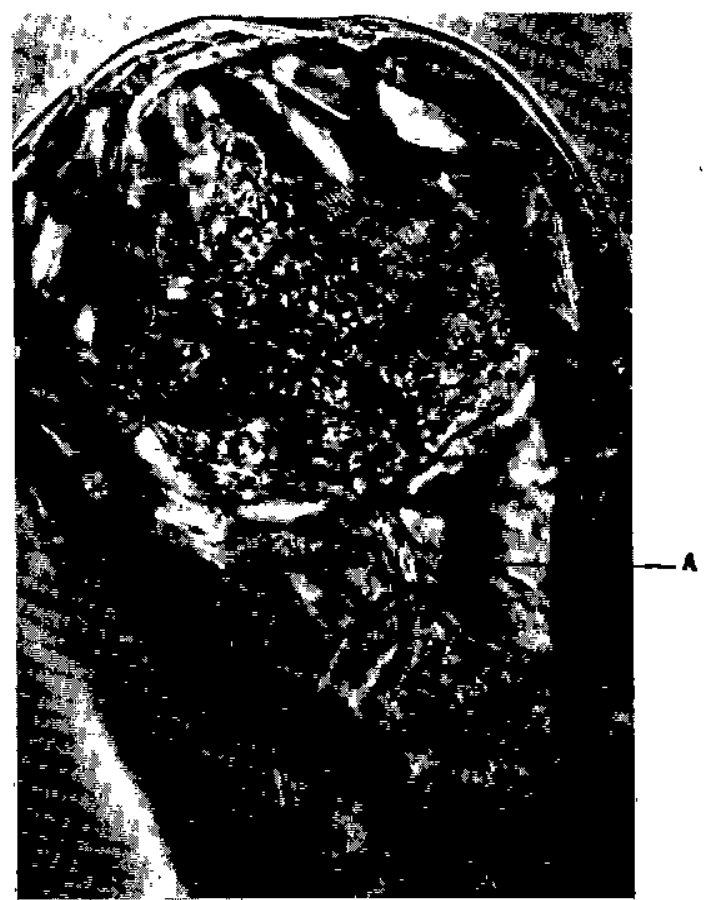

A : Gouttière dorsale $(\times 500)$. Fig. 4. 
Toujours sur le bord antérieur, mais en position dorsale (Pl. 1, fig. 3), il existe une incision en forme d' $U$, de $24 \times 24 \mu$, dont les branches supérieures portent 2 petites dents dorsales faiblement saillantes.

A l'intérieur de la capsule buccale, prennent place :

- Dans la région dorsale, directement dans le prolongement de l'incision en $\mathrm{U}$, une gouttière (Pl. 1, fig. 2) qui renferme le canal de la glande csophagienne ;

- Deux lancettes sub-ventrales.

L'œsophage, dilaté dans sa partie postérieure là où il rejoint l'intestin et où se trouvent les trois valvules habituelles, a une longueur de 1,4-1,5 mm chez le mâle et de 1,5-1,6 mm chez la femelle. Dans sa partie antérieure, on note la présence d'épaississements asophagiens fortement réfringents et disposés sur une seule ligne.

Les deirides sont bien développées, coniques et situées légèrement en arrière du milieu de l'œsophage. L'anneau nerveux est un peu plus antérieur.

Chez le mâle, la bourse caudale a la disposition classique des ancylostomidés : elle est trilobée avec un lobe dorsal peu marqué.

La côte dorsale (Pl. 2, fig. 2) est bifide à son extrémité distale et chacune de ses branches est tridigitée.

Les côtes externo-dorsales prennent naissance sur la dorsale, non loin de sa base. Les côtes latérales sont issues d'un tronc commun (Pl. 2, fig. 1). Les postéro et médio-latérales sont

Planche 2. - Bourse caudale du mâle.

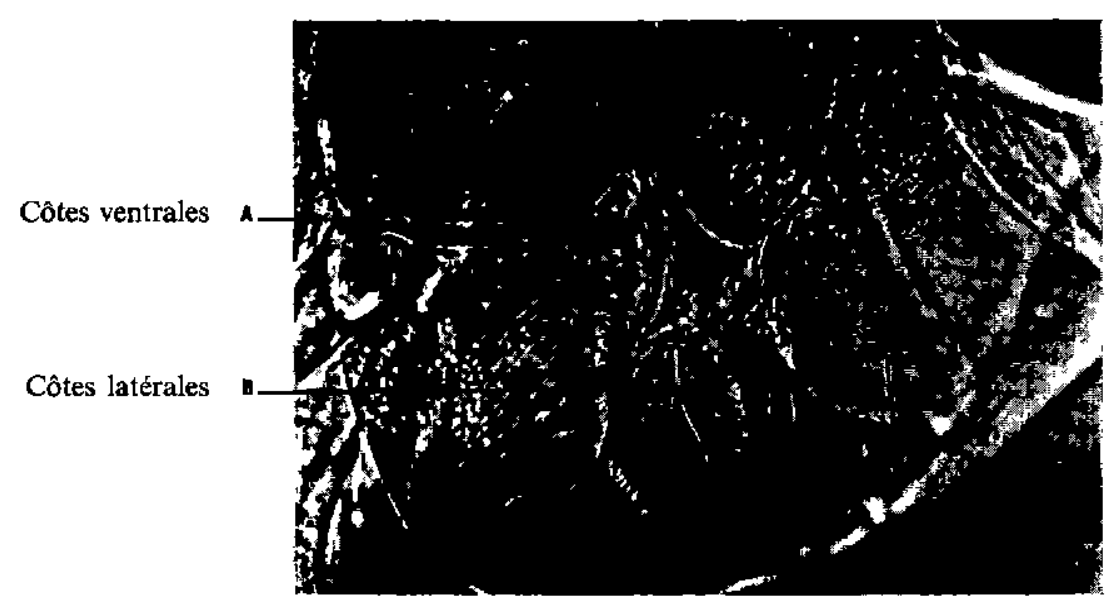

Fig. 1. - Côtes latérales et ventrales $(\times 250)$.

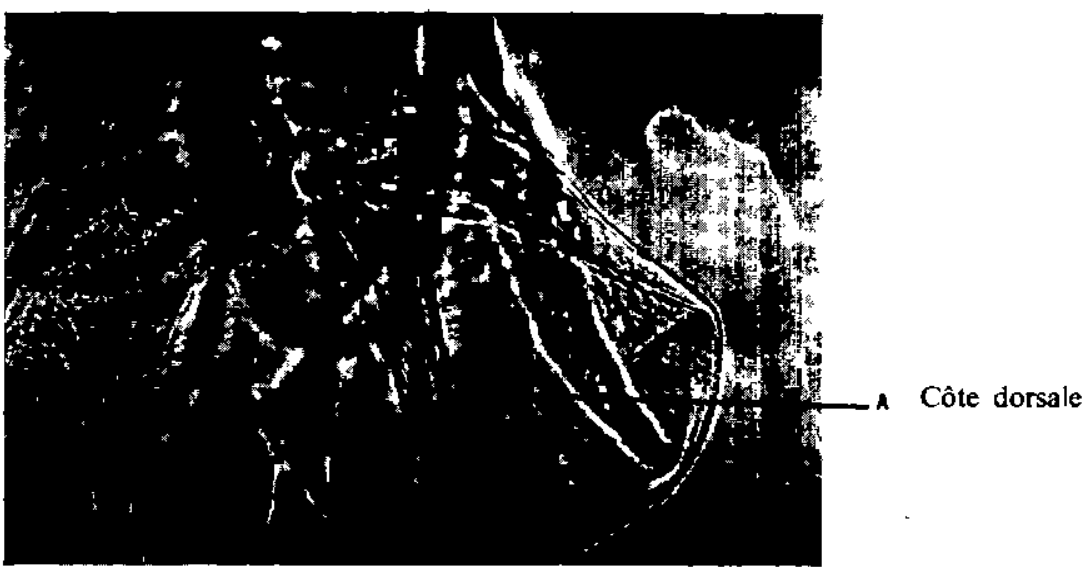

Fig. 2. - Côte externo-dorsale et Côte dorsale ( $\times 250)$. 
épaisses et droites, l'externo-latérale, plus mince et plus longue, se sépare nettement des deuxprécédentes.

Les côtes ventrales (Pl. 2, fig. 1) sont étroitement juxtaposées, la ventro-ventrale étant un peu plus longue que la ventro-latérale.

Les spicules égaux, grêles, longs de 1,4$1,5 \mathrm{~mm}$, sont soudés à leur extrémité distale qui se termine par une courte pointe. Le gubernaculum en forme de gouttière mesure $150 \mu$.

Chez la femelle, la vulve s'ouvre à $5,7-6,5 \mathrm{~mm}$ de l'extrémité caudale, soit entre le quart et le tiers postérieur. Les ovojecteurs sont pourvus de sphincters puissants. La queue, conique et longue de 180-205 $\mu$, est prolongée en général — mais ce n'est pas une règle absolue - par un mucron de $18 \mu$.

Les œufs utérins ellipsoïdes, à coque mince, mesurent 63-70 × 30-36 $\mu$ (moyenne, 66,4 $\times$ $33,6 \mu$ ). Ils renferment 2 ou 4 blastomères.

\section{DISCUSSION}

1. Parmi les ancylostomidés pourvus de 2 dents ventrales subégales, c'est-à-dire les ancylostomes du "groupe » Duodenale, plusieurs espèces s'avèrent très proches du matériel recueilli en Ethiopie :

1.1. Galoncus perniciosus Von Linstow, 1885.

Ce nématode qui vit dans des nodules creusés dans la paroi de la partie moyenne de l'intestin a été rencontré à de nombreuses reprises chez des félidés (lion, panthère, tigre) autopsiés en Afrique $(2,9,16)$, en Asie $(3,14)$ et dans divers parcs zoologiques d'Europe $(6,7,15,16,19,20)$.

Selon RAILLIET (16), Galoncus perniciosus possède 1 capsule buccale incomplète dont la paroi dorsale n'est pas chitinisée, 2 dents ventrales et 1 dent dorsale. Les lancettes subventrales font défaut, ainsi que l'incision du rebord antérieur de la capsule buccale.

Presque à la même époque, IHLE (7) redécrit le parasite : pour lui, seul ; le premier caractère retenu par RAILLIET demeure valable, car il existe bien 2 paires de dents ventrales et des lancettes sub-ventrales.

Cette opinion est suivie par la plupart des auteurs classiques (YORKE et MAPLESTONE, SKRJABIN et collab.).

Récemment, REP (17) travaillant sur des lots importants d'Ancylostoma duodenale provenant du Zaïre, constate qu'en plongeant un certain nombre d'exemplaires dans un liquide fixateur à base d'hydrate de chloral, on obtient, au niveau de la capsule buccale, des modifications telles que ces vers peuvent alors être considérés comme des Galoncus. Les différences observées entre les deux genres ne seraient que des artefacts dus au traitement que les parasites subissent avant examen.

Ce point de vue est indirectement confirmé par BAER (2) qui, dans sa description de Galoncus perniciosus, ne mentionne pas l'absence de chitinisation d'une partie de la capsule buccale.

Dans ces conditions (17), le genre Galoncus devrait être mis en synonymie avec le genre Ancylostoma et Galoncus perniciosus, de par ses caractères morphologiques et ses dimensions, serait, en réalité, Ancylostoma duodenale.

1.2. Ancylostoma paraduodenale Biocca, 1951, également parasite de divers félidés sauvages de la faune africaine (serval, lion, Guépard) en Somalie et en Zambie (4).

Par rapport à Ancylostoma duodenale, Ancylostoma paraduodenale est de plus petite taille (ơ, 5-8 mm; $96,5-8,5 \mathrm{~mm})$. La « troisième dent ventrale rudimentaire interne » peut ou être absente ou constituer une véritable dent. L'incision du rebord dorsal de la capsule buccale est arrondie et beaucoup plus réduite. Les côtes latérales de la bourse caudale du mâle n'ont pas les mêmes angles et la queue de la femelle est aplatie dorso-ventralement.

REP (17) estime qu'en raison des variations importantes existant dans une même population d'Ancylostomes, les différences observées sont minimes et qu'elles ne permettent pas de distinguer sûrement Ancylostoma duodenale d'Ancylostoma paraduodenale qui doit être mis en synonymie.

1.3. Ancylostoma malayanum Alessandrini, 1905, parasite de l'homme et de divers animaux sauvages dans le sud-est asiatique. C'est un ancylostome de grande taille dont les spicules atteignent $3 \mathrm{~mm}$ de long. REP (17) en fait, d'ailleurs, une variété d'Ancylostoma duodenale.

1.4. Ancylostoma duodenale galagoi, découverts chez un galago du Zaïre (Shaba). VAN DEN BERGHE (19) hésite à assimiler complètement cette espèce à Ancylostoma duodenale du fait de sa petite taille $(\not, 7,75 \mathrm{~mm})$, de la longueur des spicules $(1,4 \mathrm{~mm})$ et de la présence d'une "troisième dent rudimentaire interne" sur la paire médiane des dents ventrales, caractère qui, selon REP (17) est inconstant (voir supra), difficile à mettre en évidence, ce qui ne 
justifie pas la création d'une nouvelle espèce ou d'une nouvelle sous-espèce.

Le même raisonnement vaut pour Ancylostoma mephitis Micheletti, 1929 dont l'hôte est, en Afrique, le zorille (12).

1.5. Quelle que soit la position que l'on adopte à l'égard des idées de $\operatorname{REP}(17,18)$, il est bien évident que l'ancylostome de l'hyène d'Ethiopie n'est ni Galoncus perniciosus, ni Ancylostoma paraduodenale, ni Ancylostoma malayanum, ni Ancylostoma duodenale galagoi.

Ancylostoma duodenale est l'espèce qui s'en rapproche le plus tant par ses caractères morphologiques (capsule buccale, dents ventrales, gouttière dorsale, aspect de la bourse caudale du mâle et de la queue de la femelle) que par ses dimensions qui sont comprises dans les limites données par REP $(17,18)$.

2. A de nombreuses reprises, la présence d'Ancylostoma duodenale a été signalée chez des mammifères autres que l'homme, à savoir :

- Divers singes en Afrique et en Asie: gorille, chimpanzé, gibbon, macaque $(17,19)$.

- Des viverridés : civette, genette au SierraLeone (1) et aux Indes (3).

- Des félidés : tigre (3) ; lion mort en captivité (19).

- Des mustélidés : zorille (12).

- Des canidés : fennec autopsié au jardin zoologique de New York (11).

Ancylostoma duodenale affecte également le chien (19). Des essais d'infestation expérimentale (13) ont été couronnés de succès. Dans cette espèce, la durée de la phase prépatente est de 5 semaines.

- Des ongulés suiformes : porc (19).

L'hyène tachetée est un hôte nouveau.

3. Ces mammifères réagissent différemment à l'infestation par les ancylostomes du groupe "duodenale ".

3.1. Le chien et l'hyène paraissent être des hôtes normaux et les ancylostomes dont ils sont porteurs ne peuvent être différenciés d'Ancylostoma duodenale.

3.2. Par contre, la plupart des grands fauves (lion, tigre, panthère) se comportent comme des hôtes défavorables : les ancylostomes ne demeurent pas dans la lumière de l'intestin, mais pénètrent à l'intérieur de la paroi en provoquant la formation de nodules réactionnels de la grosseur d'un pois qui renferment, outre les para- sites, une masse nécrotique plus ou moins purulente.

Ces nodules, parfois fort nombreux, sont mal supportés et entraînent, en général, la mort de l'animal. C'est ce que l'on observe dans la galoncose.

3.3. Dans d'autres cas (civette, chat sauvage, guépard, quelquefois lion et tigre), le parasite évolue de façon classique. Il gagne l'intestin grêle à la surface duquel il s'accroche grâce à sa capsule et à ses dents. Cependant, l'hôte étant inhabituel, le nématode, bien que sexuellement mûr, n'atteint pas son complet développement, ce qui se traduit par une diminution de la longueur du parasite $(3,9)$. Le cas du lion dont fait état LE ROUX (10) est, à cet égard, assez typique : en effet, ce félidé hébergeait à la fois des ancylostomes de petite taille dans la lumière de l'intestin et des ancylostomes de type Galoncus beaucoup plus longs logés dans les nodules de la paroi.

4. En Ethiopie, des enquêtes effectuées à l'Hôpital d'Harrar (Harrarghé) ont montré que l'ancylostomose humaine à Ancylostoma duodenale était fort répandue, avec des taux d'infestation élevés : de 20 à 30 p. 100 des sujets examinés $(5,8)$.

Par ailleurs, l'autopsie d'une dizaine de hyènes sacrifiées dans les Provinces du Harrar, du Shoa et du Sidamo n'a permis d'isoler Ancylostoma duodenale qu'une seule fois, l'animal atteint n'étant porteur que d'un très petit nombre de parasites.

Dans l'état actuel de nos connaissances, il est difficile de tirer des conclusions définitives. Toutefois, il est permis de penser que l'hyène ne joue, en Ethiopie, qu'un rôle secondaire dans l'épidémiologie de l'ancylostomose humaine. Vraisemblablement, la contamination de cet animal se produit accidentellement, dans les régions fortement infestées comme le Harrar, à proximité des rares points d'eau qui, en saison sèche, sont fréquentés par des porteurs humains d'ancylostomes et par un grand nombre d'animaux domestiques ou sauvages.

\section{CONCLUSIONS}

Au cours de l'autopsie d'une hyène tachetée, Crocuta crocuta, effectuée dans la moyenne vallée de l'Awash, Province du Harrarghé (Ethiopie), des ancylostomes ont été recueillis dans l'intestin grêle, accrochés à la paroi de la muqueuse. 
Ses caractères morphologiques et ses dimensions permettent de rapporter ce Nématode à Ancylostoma duodenale (Dubini, 1843) Creplin, 1843 dont l'hôte habituel est l'homme.
Jusqu'à plus ample informé, l'hyène, hôte nouveau, paraît en Ethiopie, n'intervenir qu'accidentellement dans l'épidémiologie de l'ancylostomose humaine.

\title{
SUMMARY
}

\begin{abstract}
Ancylostoma duodenale (Dubini, 1843) Creplin, 1843 (Nematoda: Ancylostomidae), parasite of spotted hyaena, Crocuta crocuta (Erxleben, in Ethiopia
\end{abstract}

The nematode recovered from the small intestine of a spotted hyaena killed in Harrarghé (Ethiopia) may be referred to Ancylostoma duodenale (Dubini, 1843) Creplin, 1843.

It seems, until we have better informed, that spotted hyaena, a new host. breaks accidentally in epidemiology of human ancylostomiasis.

\section{RESUMEN}

\author{
Ancylostoma duodenale (Dubini, 1843) Creplin, 1843 (Nematoda : Ancy- \\ lostomidae) parasito de la hiena moteada, Crocuta crocuta (Erxleben) en \\ Etiopia
}

Se recogieron algunos ejemplares de Ancylostoma duodenale (Dubini, 1843) Creplin, 1843 en el intestino de una hiena moteada, matada en la provincia del Harrarghe (Etiopia).

Dicho carnívoro, huésped nuevo, no parece ocurrir más que casualmente en la epidemiologia de la ancilostomosis humana.

\section{BIBLIOGRAPHIE}

1. ADLER (S.). Ancylostomes in animals in Freetown Ann. trop. Med. Parasit., 1922, 16 (3) : 293-294.

2. BAER (J. G.). Helminthes parasites. Explor. Pares natn. Congo Belge, Miss. J. G. Baer-W. Gerber, 1959, 1 : $132-134$

3. BAYLIS (H. A.), DAUBNEY (R.). Report of the parasitic Nematodes in the collections of the zoological survey of India. Mem. Indian Mus., 1918-1922, 7 (4) : 263-347.

4. BIOCCA (E.). On Ancylostoma paraduodenale a new species from Felines, closely related to Ancylostoma duodenale. J. Helminth., 1951, 25 (1/2) : 11-18.

5. BLAHOS (J.), KUBASTOVA (B.). The survey of 11170 patients treated in the Ras Makonnen hospital in Harrar. Ethiopian Med. J, 1963, 1 : 190-196.

6. COHN (L.). Uncinaria perniciosa (Von Linstow). Archs. Parasit., 1899, 2 (1) : 5-22.

7. IHLE (J. E. W.). Ueber Ancylostoma perniciosum und die Strongyliden des Elefanten. Bijdr. Dierk., 1919, 21 : 97-103.

8. JUNK (K.). Zur epidemiologie der lepra in Sud-ost aethioplen in Zentralen hochland von Harrar. Dissertation, Hamburg, 1969.

9. LANE (C.). Ancylostoma duodenale as a parasite of Felis tigris Indian. J. med. Res., 1917, 5 (1) : 210-216.

10. LE ROUX (P. L.). Pharyngostomum cordatum (Dies., 1850), Galoncus perniciosus (Von Linstow, 1885) and Gnathostoma spinigerum Owen, 1836, infections in a lion in Northern Rhodesia. Trans. r. Soc, trop. Med. Hyg., 1958, 52 (1) : 14.

11. MCCLURE (G. W.). Nematode parasites of Mammals with a description of a new species Wellcomia branickii, from specimens collected in the New York zoological Park. Zoologica, N. Y., 1932, 15 (1) : 1-29.
12. MICHELETTI (E.). Intorno ad una nuova specie di Ancylostoma. Annali Med. nav. colon., 1929, 35 : 246257.

13. NAGAHANA (M.), YOSHIDA (Y.). Complete development and migratory route of Ancylostoma duodenale in young dogs. Parasitology, 1965, 51 (2, Sect. 2) : 52.

14. PATNAIK (M. M.), RAO (A. T.), ACHARJYO (L. N.). Notes on the nodular disease of the intestine of lion (Panthera leo) caused by Galoncus perniciosus (Ancylostomatidae: Nematoda). Indian vet. J., 1971, 48 (10) : 1072-1073:

15. PERRY (M. H.). Tumours of gut wall due to Galoncus perniciosus showing relation to helminth infections to bacterial invasion of the tissues. Proc. r. Soc. Med., 1921, $14: 23-24$.

16. RAILLIET (A.). Sur un Strongylidé vivant dans les kystes intestivaux chez les grands Félidés. Bull. Soc. Path. exot., 1918, 11 (2) : 86-93.

17. REP (B. H.). On the polyxenia of the Ancylostomidae and the validity of the characters used for their differentiation. Trop. geogr. Med., 1963, 15 (2) : 173-218 et (3) : 271-316.

18. REP (B. H.). A new system for the diagnosis of $A n c y$ lostomidae, especially the human hookworm species. Trop. geogr. Med., 1964, 16 (4) : 354-369.

19. VAN DEN BERGHE (L.). On the occurrence of a species of Ancylostoma closely related to Ancylostoma duodenale in an African Lemur. Parasitology, 1936, 22 (2) : 224-226.

20. VON LINSTOW (O.). Beobachtungen an bekannten und neuen Nematosen uns Trematoden. Arch. Naturg., 1885, 1 : 235. 